\title{
Cytokines and Hematology
}

\author{
Shinpei Kasakura
}

\author{
Kobe City General Hospital, Kobe, Japan
}

Received April 6, 2001; accepted April 10, 2001

Int J Hematol. 2001;74:1-2.

(C)2001 The Japanese Society of Hematology

As of today, more than 50 cytokines have been identified, and more cytokines and receptor molecules will continue to be discovered at a good pace through searches for sequence homology in expressed sequence-tag databases [1].

Through research on cytokines over the past 40 years, a tremendous amount of information has emerged regarding the structures of cytokines and cytokine receptors, their signal transduction systems, their production mechanisms, their biological functions, and their role in human disease. Therapeutic approaches have been explored that use either exogenous cytokines for insufficient cytokine activity or anticytokine reagents (such as monoclonal antibodies [MoAbs] and soluble receptors) for excessive cytokine activity. The levels of cytokines in serum and of cytokine messenger RNA (mRNA) in tissue can be measured and used as diagnostic and prognostic markers for human disease.

However, some important problems remain to be solved. The cytokine network is a redundant system in which a single cytokine may mediate many effects and several cytokines may exert only a single effect. Due to the redundancy of the cytokine network, we still understand very little about the exact roles played by each cytokine in either the regulatory or the effector mechanisms in normal and disordered physiology. It seems to be pertinent to assume that inhibiting a single cytokine allows another cytokine to become up-regulated and act as a substitute, performing the original cytokine's function in the cytokine network. Advances in our understanding of such characteristics of the cytokine network can lead to development of useful therapeutic reagents for treating human disease.

A historical account of the discovery and development of cytokines is reviewed comprehensively by Joost Oppenheim in this issue of the International Journal of Hematology [2]. Oppenheim, who is one of the pioneers of cytokine research, also describes what is understood about cytokines in light of recent discoveries. Finally, he foresees a long-term future for the discipline of "cytokinology" as a biomedical science.

Chemokines were initially identified as leukocyte chemoattractants that induce cellular movement from the circulatory system into inflamed tissue. As of today, more than 50 chemokines and 18 chemokine receptors have been identified. We now know that chemokine function extends far beyond leukocyte migration. CC family chemokines regulate immune responses through the recruitment and activation of $\mathrm{CD}^{+}{ }^{+} \mathrm{T}$ lymphocytes. Stromal cell-derived factor 1 , a CXC chemokine, exerts a chemoattractant effect on early hematopoietic cells [3]. Recent studies also provide evidence that chemokines play a role in hematopoiesis. Broxmeyer and Kim [4] have found that numerous chemokines exert suppressive activity on proliferation of hematopoietic stem/progenitor cells, identifying the chemokines that suppress hematopoiesis as MIP- $1 \alpha$, Gro- $\beta$, PF4, IL-8, MCP-1, and IP-10. In this issue of the Journal, Hal Broxmeyer reviews chemokines and chemokine/receptor activities mainly in the context of hematopoietic cell regulation [5].

Transforming growth factor $\beta$ (TGF- $\beta$ ) mRNA and its receptors are expressed in a wide variety of tissues and cells, suggesting that TGF- $\beta$ mediates pleiotropic effects on multiple cell types. TGF- $\beta$ regulates proliferation and differentiation of cells, embryonic development, bone remodeling, wound healing, and angiogenesis. TGF- $\beta$ is also important in hematopoiesis, inflammation, and immune response [6]. Ruscetti et al have energetically studied the role of TGF- $\beta$ in hematopoiesis and they have found that TGF- $\beta$ has potent hematopoietic regulatory properties that can either inhibit or enhance the growth and differentiation of hematopoietic progenitor cells [7]. In this issue of the Journal, Frank Ruscetti and Stephen Bartelmez describe details on the role of TGF- $\beta$ in the regulation of stem cells and committed progenitor cells [8]. Increases or decreases in the production of TGF- $\beta$ may be linked to hematological diseases.

Graft-versus-host disease (GVHD) is the most important complication of allogeneic hematopoietic stem cell transplantation. The cytokine cascade is believed to cause many of the effects of GVHD [9]. However, details regarding the interaction of cytokines and the exact role of each cytokine in the cytokine cascade leading to GVHD have not been completely elucidated. Interleukin (IL)-2 was considered to be critical early in the development of GVHD. Cyclosporin A, an agent that inhibits IL-2 production, has been shown useful in clinical prophylaxis of GVHD. Inhibition of tumor necrosis factor (TNF)- $\alpha$ with anti-TNF- $\alpha$ MoAb can prevent GVHD in experimental GVHD models. However, the phase I-II clinical trials with a humanized anti-TNF- $\alpha$ MoAb for 
the treatment of acute GVHD have resulted in limited success. Recent studies suggest that acute GVHD is associated with an overproduction of type 1 cytokines such as IL-2, interferon $\gamma$, and IL-12, whereas chronic GVHD is characterized by the overproduction of type 2 cytokines such as IL-4 and IL-10. T-helper cell (Th)1/Th2 imbalance seems to play a critical role in the induction of acute versus chronic GVHD. The agents that modulate Th1/Th2 balance to downregulate Th1 or Th2 are under investigation for use in treatment of GVHD. In this issue of the Journal, Joachim Deeg comprehensively describes the role and interaction of all cytokines involved in the pathogenesis of GVHD [10].

\section{References}

1. Adams MD, Kelley J M, Gocayne JD, et al. Complementary DNA sequencing: expressed sequence tags and human genome project. Science. 1991;252:1651-1656.

2. Oppenheim JJ. Cytokines: past, present, and future. Int J Hematol. 2001;74:3-8.

3. Aiuti A, Webb JJ, Bleul C, Springer T, Gutierrez-Ramos JC. The chemokine SDF-1 is a chemoattractant for human CD34+ hematopoietic progenitor cells and provides a new mechanism to explain the mobilization of CD34+ progenitors to peripheral blood. $J$ Exp Med. 1997;185:111-122.

4. Broxmeyer HE, Kim CH. Regulation of hematopoiesis in a sea of chemokine family members with a plethora of redundant activities. Exp Hematol. 1999;7:1113-1123.

5. Broxmeyer HE. Regulation of hematopoiesis by chemokine family members. Int J Hematol. 2001;74:9-17.

6. Letterio JJ, Roberts $\mathrm{AB}$. Regulation of immune responses by TGF- $\beta$. Annu Rev Immunol. 1998;16:137-161.

7. Ruscetti FW, Jacobsen SE, Birchnall Roberts M, et al. Role of transforming growth factor-beta 1 in regulation of hematopoiesis. Ann N Y Acad Sci. 1991;628:31-43.

8. Ruscetti FW, Bartelmez SH. Transforming growth factor- $\beta$ : pleiotropic regulator of hematopoietic stem cells: potential physiological and clinical relevance. Int J Hematol. 2001;74: 18-25.

9. Vogelsang GB, Hess A. Graft-versus-host disease: new directions for a persistent problem. Blood. 1994;84:2061-2067.

10. Deeg HJ. Cytokines in graft-versus-host disease and the graft-versus-leukemia reaction. Int J Hematol. 2001;74:26-32. 